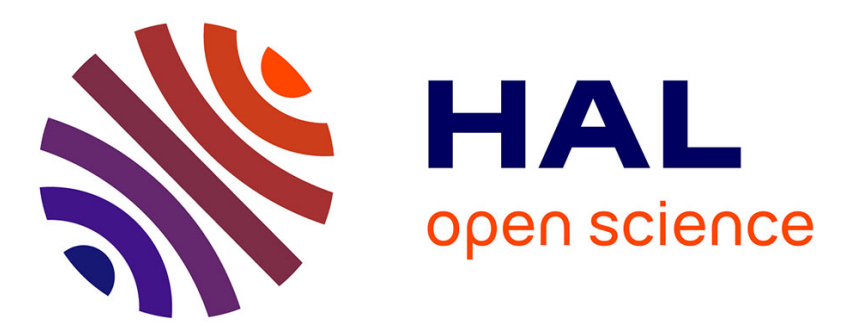

\title{
Autonomic Renumbering in the Future Internet
}

\author{
Frédéric Beck, Isabelle Chrisment, Ralph Droms, Olivier Festor
}

\section{To cite this version:}

Frédéric Beck, Isabelle Chrisment, Ralph Droms, Olivier Festor. Autonomic Renumbering in the Future Internet. IEEE Communications Magazine, 2010, 48 (7), pp.86-92. 10.1109/MCOM.2010.5496883 . inria-00531215

\section{HAL Id: inria-00531215 https://hal.inria.fr/inria-00531215}

Submitted on 2 Nov 2010

HAL is a multi-disciplinary open access archive for the deposit and dissemination of scientific research documents, whether they are published or not. The documents may come from teaching and research institutions in France or abroad, or from public or private research centers.
L'archive ouverte pluridisciplinaire HAL, est destinée au dépôt et à la diffusion de documents scientifiques de niveau recherche, publiés ou non, émanant des établissements d'enseignement et de recherche français ou étrangers, des laboratoires publics ou privés. 


\title{
Autonomic Renumbering in the Future Internet
}

\author{
Frédéric Beck, Isabelle Chrisment, Ralph Droms and Olivier Festor
}

\begin{abstract}
IPv6 is an essential building block of the evolution towards the future Internet. To take the full benefit of this protocol and exploit all its features, the future Internet needs to gracefully couple it with autonomics. In this paper we demonstrate through our experience with network renumbering how the coupling of both IPv6 core functionality extended with major functions of the autonomic world can lead to fully autonomous activities of main management functions. We instantiate the notions of self-configuration, self-monitoring, selfprotection and self-healing in the network renumbering process and show how they can altogether make renumbering a real success. We illustrate the various functions with the tools we implemented to support them over the last three years.
\end{abstract}

Index Terms - Autonomics, IPv6, Management, Renumbering

\section{INTRODUCTION}

Designing the building blocks of the Future Internet is today one of the most important challenge for the networking community. Visions are flourishing, encouraged by many large initiatives like GENI ${ }^{1}$ in the USA and the Future Internet initiative ${ }^{2}$ part of the European $7^{\text {th }}$ framework program.

Two main approaches have emerged as the leading paths to the future Internet: one advocating a clean slate, revolutionary approach to design the future Internet and a second one advocating an evolutionary approach extending current protocols with the required capabilities to address all issues of the future Internet.

In this paper we follow the evolutionary path and address one specific management function enabled by the IPv6 protocol namely renumbering. Network renumbering is a very interesting feature of IPv6 which offers very promising perspectives in terms of automated network adaptation through self-configuration; it is also one of the most risky procedures, which needs a special attention in the management plane. Despite a decade of standardization, this essential function still needs work as reaffirmed recently by Carpenter et al. in [10]. To enable full automation of this function, several self-management functions have to be added to the network to complement the basic functionality offered by the IPv6 protocol for this task, mostly advertising. As described in [9], self-management involves the interaction of five functional blocks :

- Self-configuration at device level and SelfOrganization at a network level to adapt configuration to context.

- Self-protection to recognize and avoid threats (DDOS, heat, power failure).

http://geni.net/

http://www.future-internet.eu/
- $\quad$ Self-healing to diagnose abnormal operation and take actions to normalize the behavior.

- Self-optimization to continuously improve the system performance.

- Self-monitoring to continuously collect state and context information.

Additional strata, often transversal to the above listed functions are commonly agreed-upon in the autonomic networking communities nowadays like network awareness [11], which extended with self-discovery and analysis features leads to autognostics.

While the network renumbering service is enabled by the neighbor discovery protocol built in IPv6, we show in this paper that without adding the previously listed functions to the management plane, renumbering will not guarantee the integrity and safety of the concerned network. In addition to motivating the support provided by autonomic features in the renumbering process, we describe the services that can support them and show through the tools we built over the last couple of years, how to aid IPv6 network renumbering to become really autonomic and thus an essential building block of the future Internet.

The paper is organized as follows. Section II is dedicated to the renumbering service and self-configuration issues linked to it. The problems that arise if the function is not addressed in a holistic way are also presented in this section. Section III addresses the self-monitoring part of the solution that leads to an autonomic IPv6 renumbering. Self-protection and selfhealing in network renumbering are the subject of section IV. Section V contains a synthetic view of the tool support that can cover the self-functions for IPv6 renumbering. Section VI concludes the paper and draws some directions for future work.

\section{SELF-CONFIGURATION AND RENUMBERING}

IPv6 network renumbering is a procedure in which all devices located on a subnet change their IPv6 prefix address. The effects of renumbering can be reduced by replacing an old prefix by a new prefix without a "flag day" permitting a period of time where both prefixes are in use. Services transit from the old prefix to the new prefix by simultaneously using the old prefix for existing service connections while using the new prefix for any new service invocation. The first function to ease this feature is to automate IP address configuration. In this section, we show how the self-configuration building block is applied through the Neighbor Discovery Protocol [2]. We also point out that self-configuration does not avoid some problems, which can lead to the failure of the renumbering procedure. 


\section{A. IPv6 network renumbering}

Renumbering can be triggered by many different events like changes in the internal topology, a network merge, uplink prefix changes due to the migration towards a new provider or to a dial on demand. The frequency of these events varies and can complicate the task of the administrator. Several investigations have already been made on this topic within numerous projects like for instance $6 \mathrm{net}^{3}$ in Europe. The major challenge is to achieve transparent renumbering and avoid disruptions for the users. To reach this aim, a procedure in eight steps has to be followed [4]:

1. Stable and working situation with an existing prefix (old prefix).

2. Obtain the new prefix and new reverse zone from the delegating authority.

3. Set up a parallel routing architecture for the new prefix.

4. Hosts' addressing: the new prefix is announced.

5. Stable configuration where the network is multihomed (with two or more addresses). IPv6 explicitly allows multiple prefixes to be assigned to a link simultaneously.

6. Old prefix is obsolete (lifetimes set to zero). The transition from the old to the new prefix for services can be done.

7. Remove the old prefix. Addresses for the old prefix are deleted from the hosts' interfaces.

8. Equivalent to the first state, but using the new prefix.

One cause of renumbering is a change in the network numbering architecture, which results from moving to a new service provider. Multi-homing in IPv6 is a useful feature to minimize the impact a renumbering event, but is not sufficient to solve the renumbering issue. Multi-homing enables multiple connections to different providers but has important issues to be dealt with like ingress filtering or induced explosion of routing tables sizes. When a network is renumbered, the multihoming phase is used for a very limited time (as short as possible) as the IPv6 prefix has to be changed.

Anycast can also be considered as a useful service to ease renumbering. This however does not work since either anycast addresses are taken from the unicast address space and as such depend on renumbering or if their prefix is not bound to the unicast space, they must be announced as a separate route and thus lead to an unwanted increase in routing tables. Even if it would be useful on a single site, its use for hosts address assignment is prohibited in RFC 4291 [12].

The IPv6 Neighbor Discovery Protocol is used to announce the assignment of IPv6 prefixes in a network, replacing IPv4 Router Discovery [7] and ARP [8]. It also adds new functionalities such as IPv6 Stateless Address Autoconfiguration [3]. Neighbor Discovery allows a network administrator to specify the prefixes assigned to a link and enables a node to automatically configure the addresses and routes, simply by being connected to the network link.

\footnotetext{
$3 \quad$ http://www.6net.org
}

As illustrated in Figure 1, the Router Solicitation (RS) message is sent by a node to ask for information about the onlink routers and prefixes. The router replies with a Router Advertisement (RA) providing the default gateway's address, the router's validity, the list of IPv6 prefixes the router handles, Maximum Transfer Unit (MTU), Mobile IPv6 options and several other informations. Additionally, the router can send an unsolicited RA message, which can be used to announce new prefixes or deprecate existing prefixes, as in steps 4 and 6 of the renumbering procedure. Using RS and RA messages, a node can dynamically configure addresses and other information about its interface.

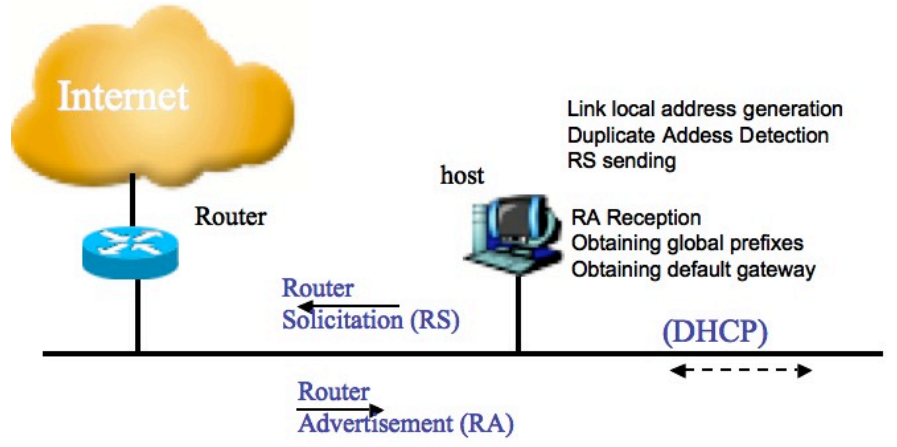

Fig. 1. Auto-configuration mechanism

A host using the stateless auto-configuration facility has to control whether the obtained address is already used in the network through a duplicate address detection mechanism. If stateful auto-configuration is preferred, a DHCP server will be contacted.

\section{B. Issues}

To successfully complete the renumbering process, more changes are required in both nodes and routers including:

\section{1) Manually configured hosts/hard coded addresses}

Addresses often appear to be "hard coded" parameters in configuration files and even in some applications'source code. These addresses thus have to be manually updated to move from the old prefix to the new prefix because address autoconfiguration will not be performed on these services when a new prefix is announced. After such a modification, the service or the tool must usually be restarted to reload the proper configuration file. This can lead to situations where the host becomes unreachable and then, all the associated services will be down.

\section{2) $D N S$}

As hosts renumber their interfaces, their corresponding resource records in the DNS database must be updated. Specifically, as the new prefix is enabled in step 4 of the renumbering process, the new IPv6 addresses assigned by each host must be added to the hosts' IPv6 records (type AAAA). Similarly, as the old prefix is deprecated in step 6, the addresses from the old prefix must be removed from the hosts' AAAA records. The TTL (time to live) for the deprecated addresses must be coordinated so that these addresses expire from DNS caches at the appropriate time. 
Similarly, problems can appear for services that make a single resolution (usually at startup), or for applications that bind to a specific address that is subsequently modified during renumbering. In such cases, the service or application must be restarted to become operational again after renumbering.

\section{3) Data continuity problems}

Some monitoring applications store information on the evolution of a host in order to update statistics. After renumbering, if monitored hosts are not properly identified (e.g. when the address is used for this purpose), data continuity problems arise.

\section{4) Security}

When the routing architecture for the new prefix is being set up, the network parts running the routing protocol are vulnerable to attacks, as the Access Control Lists (ACLs) are not set for the new prefix yet. For example, when routing is activated on the DMZ and the servers renumbered, the web or mail servers will still be protected against illegal access for the old address, but if ACLs for the new address are not set at the right time, the servers become vulnerable for this newly assigned address. The routing infrastructure itself can be compromised, even before the hosts are renumbered. When routing is activated for the new prefix, the routers have already assigned addresses corresponding to the new prefix. Thus, they are in the same situation as the servers mentioned in the previous example, and are likely to suffer various types of attacks or intrusions. To protect them, the first thing to do before advertising the prefix is to update both ingress and egress access lists by including rules for the new prefix.

Given the many specific cases in which renumbering affects network operation that are not solved by automated address assignment, self-configuration is not sufficient to maintain a coherent and operational network through a renumbering event. In order to prevent as many problems as possible, the network administrator has, prior to finalizing the renumbering, to achieve some tasks like performing the renumbering of all the routers and switches or adjusting the prefixes' lifetimes in router advertisements, DHCP leases, DNS entries validity in caches... To automate these operations, a strong coordination among the entities is required and a global view of the network is necessary so as to adapt the configuration to the global policy of the organization.

\section{SELF-MONITORING}

IPv6 network renumbering is a complex procedure that has to follow several steps. As shown in the previous section, applying self-configuration is only part of the renumbering process. Each step must be successful and validated before moving to the next one. Following the procedure manually is fastidious, especially when the renumbered network is composed of an important number of end-hosts. So a tight connection between self-configuration and self-monitoring is required. Through self-monitoring, a network view is continuously provided and allows an autonomic manager to orchestrate and optimize the whole procedure.
The autonomic manager collects information about individual hosts, and can infer the global state of the renumbering procedure as well as the state of services running on the renumbered network to ensure that those services are functioning properly as the renumbering takes place. This information can be collected from an agent on each host which communicates with a renumbering manager, as described in section V. Based on the manager's knowledge of the preconditions and post-conditions of each step in the renumbering procedure, the autonomic manager can monitor the progress through each renumbering step and validate the transition from each step to the next. In the occurrence of problems or because of local policies, the autonomic manager can request operator intervention and/or administrator confirmation before proceeding to the next step.

Self-monitoring becomes especially important in step 5 of the renumbering procedure. In this step, the network is in a stable situation where both the old and new prefixes are available. This step marks the beginning of the transition phase between the two prefixes. The transition phase ends when all devices stop using the old prefix for both their outgoing and incoming communications. Self-monitoring helps to determine exactly the end of this transition, ensuring that all the hosts do not use the old prefix anymore thus avoiding service outage. Self-monitoring optimizes the transition phase duration and contributes to reduction in cost of the maintenance of both Internet connections by enabling the old one to be released earlier.

During the whole procedure, the autonomic system must check that the running services on the network remain accessible in accordance with the procedure. For example, during steps 1 to 4 , a Web server should answer only to its old address, whereas during steps 5 to 7 , until the old address is suppressed, it should respond to both addresses, and finally in step 8 only work with the new address. Self-monitoring allows the participating entities to test among themselves the availability of the services and to ensure the accessibility of services before validating the transition to a new step. This monitoring can be done in a fully distributed way.

Some problems can be automatically detected during the renumbering procedure since the autonomic manager infers a global state of the procedure, and orchestrates the transitions between the different steps. For example, the manager validates the transition between steps 4 and 5 once all the monitored hosts are in a multi-homing situation. If some hosts do not begin to use an address for the new prefix, it usually means that they are not using stateless auto-configuration. If they are using stateful auto-configuration with DHCPv6, the manager may trigger a reconfiguration, either by telling the DHCP server to send a reconfigure message to the host, or by triggering directly the process on the host via the monitoring agent. If the host is using static addressing, the agent may also reconfigure the addressing locally, while following the directions given by the manager. If the problem concerns a service which is not available for the new address, or any long-term session, such as SSH or NFS, which prevents the transition step to end, the system may not always be able to react automatically, as the problems are too specific or human 
related. In this case, an alert and guidelines for solving the problem should be sent to the administrator, who would then take the appropriate measures. If the issue is blocking, the autonomic system should be able to revert to a previous working and safe configuration, upon decision of the administrator.

\section{SELF-PROTECTION AND SELF-HEALING}

So far, we took the assumption that all hosts on our network were healthy and behaving as they are supposed to. In order to reach our goal of autonomic renumbering, we now have to consider some hosts on the network can be malicious. To avoid these nodes from harming the network, it must be protected, and the troubles created by malicious nodes must be corrected. This process commonly comprises both selfprotection and self-healing functions

\section{A. Self-protection}

In the context of renumbering, self-protecting means that functions in the network should detect attacks or misconfigurations that compromise the network and the renumbering procedure. Typically, an attack consists in sending a bogus Router Advertisement (RA) during steps 4, 6 or 7 of the renumbering procedure, causing trouble and errors in the addressing of devices or in their routing tables. This bogus RA may contain false lifetimes for a prefix involved in the renumbering, or a fake prefix.

As shown in Figure 2, an autonomic device should (1) detect this bogus RA, and (2) identify the source of the message. This detection can be done via a tool like NDPMon described in section $\mathrm{V}$, which uses the knowledge of the legitimate RA and routers. Another option is to use signed RAs to avoid this problem (such as defined in the Secure Neighbor Discovery SEND protocol), but this option requires the deployment of a complex architecture, including certificate authorities and usage of compliant nodes and routers. Moreover, the usage of IPSec as a building block of SEND introduces additional processing of the RA, which may be harmful for real time communications. In our architecture, we opted for the first option, because we believe that this is the situation we will encounter in most of the cases. After detecting a bogus RA, the autonomic system, via the autonomic manager, or via any other device upon decision of the manager, as shown in figure 3 , will reconfigure the network entity/ies in order to place an Access List (ACL) directly in the network component to block malicious traffic from the bogus system on the network, to stop the attack and prevent any further ones originating from the same device.

Self-protection during a renumbering procedure does not only address misconfiguration and attacks against the renumbering procedure. As this process is based on the Neighbor Discovery Protocol, all attacks against this protocol, as described in [5], have potential to harm the procedure and the network.

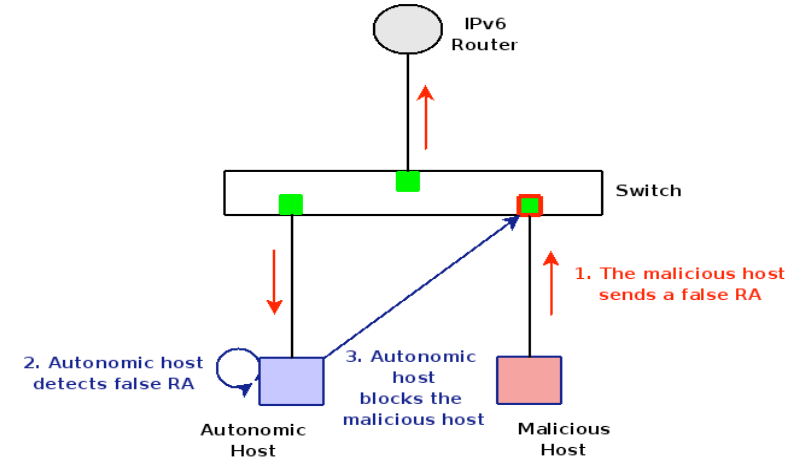

Fig. 2. Self-protection in network renumbering

\section{B. Self-healing}

Once a problem has been detected and once the attacked device has protected itself, the system must have the ability to recover from the attack either by finding an alternative way of using resources or reconfiguring itself to keep functioning smoothly.

If we keep the same example as for self-protection, the damages the network underwent must be corrected so that the network reverts to its original and valid state. As the issuance of an invalid RA was the cause of the trouble, the healing procedure consists in sending a new valid RA, which will annihilate the effects of the bogus one. If the RA contained wrong information about a valid prefix, resending the valid RA is sufficient, as shown in figure 3.

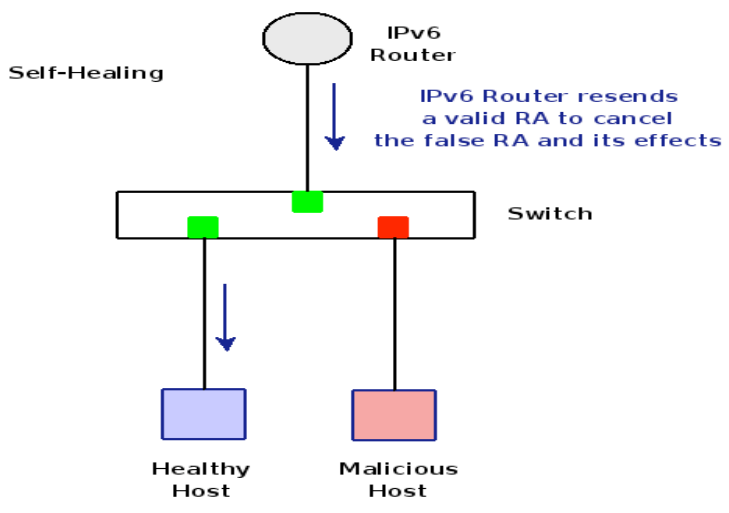

Fig. 3. Self-healing during network renumbering

If the RA did advertise a wrong prefix, we need to forge an equivalent RA, but with modified lifetimes (preferably very short ones), to ensure all traces of the wrong prefix do disappear as fast as possible.

The counter measure to be taken while performing selfhealing depends on the problem detected by the self-protection operation. These two aspects of an autonomic system are closely linked in the autonomic renumbering process.

Moreover, all problems or potential problems detected and solved should be logged, and the administrator should be warned via an alarm system. He will thus be able to validate the changes, correct the misconfiguration, and take further measures to ensure that the malfunctions do not reappear, especially if they are caused by the intrusion in the network of an attacker. 


\section{V.TOOLS SUPPORT}

All components of the autonomic renumbering engine were implemented in an integrated architecture. In this section, we present our architecture and the tools that can be combined to build the autonomic renumbering solution. We also address the necessary interoperability issues.

\section{A. Monitoring the renumbering procedure}

The monitoring part of the architecture is composed of three entities: management agents, one renumbering manager and service renumbering probes.

Monitoring agents are deployed either within systems or at strategic monitoring points. They are able to diagnose whether the monitored devices did renumber properly or, if not, why. The availability of such agents is important since it provides the necessary semantics to help control the loops to take appropriate decisions.

A manager can be physically distributed among several entities. It has in charge the validation of the renumbering triggers (router advertisement, administrators orders) and orchestrates the renumbering process.

To complement the agents and the manager, we implemented a set of probes. Their task consists in permanently monitoring the health of the services while the renumbering process is going on.

This architecture (with a simplified central manager service) is implemented in a tool called $\mathrm{NetSV}^{4}$, which provides a monitoring service for the renumbering and validates the addressing of hosts. NetSV also provides continuous diagnosis on the monitored devices by preventing and/or detecting problems that could occur during such an operation.

NetSV is divided into three elements as shown in Figure 4 :

- a daemon running on monitored devices (or remotely representing monitored devices) sending information on the local addressing to the monitoring host,

- a daemon running on the monitoring host using the information sent by the agents to maintain a global state of the renumbering procedure ;

- a diagnostic tool, independent from the other blocks, taking care of service checks and diagnostics on monitored hosts.

With this tool, an administrator can follow the whole renumbering procedure and validate each step.

\section{B. Monitoring the Neighbor Discovery Protocol}

Monitoring the devices is not sufficient. The principal renumbering triggering protocol, namely the Neighbor Discovery Protocol (NDP), also needs to be monitored. Therefore, we implemented a tool called NDPMon ${ }^{5}$, the Neighbor Discovery Protocol Monitor similar in its basic functions to the IPv4 ArpWatch ${ }^{6}$. It is in charge of monitoring the Neighbor Discovery Protocol activities and maintains up-

\footnotetext{
4 http://netsv.sourceforge.net

5 like NetSV, NDPMon is distributed freely. It can be downloaded in both source or binary form for various platforms and distributions from http://ndpmon.sourceforge.net

6 http://www-nrg.ee.lbl.gov/
}

to-date a neighbor database, which contains the correspondence between IPv6 and Ethernet addresses, alongside with a timestamp. In the same way than ArpWatch, activities and suspicious behaviors raise alerts and reports.

Alerts and reports can be sent over various transport systems such as mail and syslog. In addition to its monitoring role, NDPMon is able to detect attacks against the Neighbor Discovery Protocol, as defined in [5]. Misconfiguration, stack vulnerabilities and suspicious behaviors are well addressed in NDPMon.

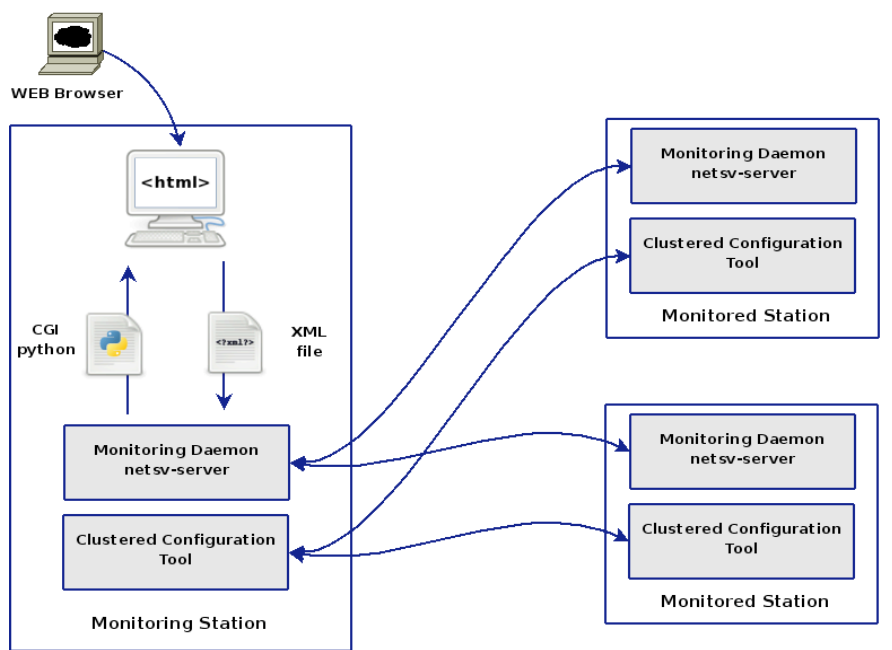

Fig 4. NetSV Architecture

NDPMon operates in two phases: a learning phase and a monitoring phase. During the learning phase, NDPMon builds the neighbors database by capturing the Neighbor Discovery messages and, based on the Router Advertisements received, it populates the routers list, while making the assumption that, when it enters the learning phase, the network is healthy. This phase is run only once, and requires close attention from the administrator. Once this phase is over, the tool can switch to the monitoring mode.

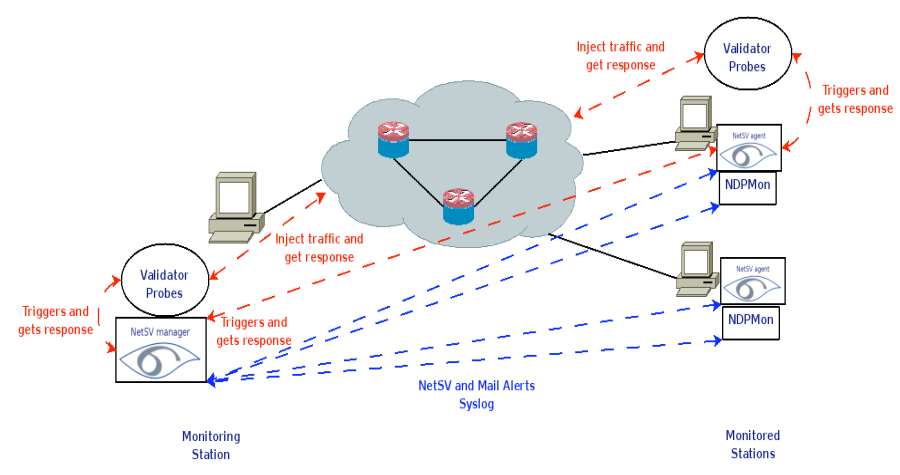

Fig 5. Self-Monitoring framework

\section{Tools interoperability}

Although NDPMon and NetSV appear to have some similarities in host monitoring functions, they are, in fact, complementary. While NetSV monitors the renumbering procedure and validates the addressing of end hosts, NDPMon 
monitors the NDP activities, and detects attacks against this protocol. Figure 5 shows how these tools can cooperate to enable full self-monitoring of a renumbering process.

For example, when the old prefix is supposed to be suppressed from advertisements, an attacker can still issue forged RA after the router which managed the old prefix stopped operating this prefix. In that case, NetSV agents will not detect the forgery of RA for the old prefix, and will not send any report to the manager. The manager will thus not detect the end of the $7^{\text {th }}$ step of the procedure. With NDPMon deployed on the network, it will send an alert to the management station, with information about the attacker (MAC, IPv6 address...), which can be used to neutralize him and activate the protection and healing functions.

\section{D.Reconfiguring the Network}

As, in our study case, the autonomic system is the network itself, configuring, protecting and healing operations mean at the end reconfiguring network equipments. Currently the platform supports Command Line Interface (CLI) configuration interfaces over Telnet or SSH and has been tested against several heterogeneous equipments. Future interfaces with Netconf [6] are foreseen once an integrated data model is available for this technology.

\section{CONCLUSIONS}

A major issue towards the acceptance of IPv6 as one of the key building blocks of the Future Internet is its capacity to offer real autonomic behavior. In this paper, we focused on enabling the fully autonomic behavior of network renumbering. Based on experience in various networks and development of distributed solutions for renumbering issues in the management plane, we have shown that to become fully autonomous, the current renumbering service must be backed with distributed monitoring, orchestration, protection and healing. To address these functions we have designed a distributed management scheme and implemented the supporting code that offers an integrated approach to the problem.

This work was illustrated with a concrete problem in one IPv6 automation feature. The architecture we developed to address the IPv6 renumbering issue, while generic in design, has not yet been extended to other application cases. As part of future work, we will investigate on how to adapt our architecture to autonomic networks architectures designed from a top-down approach like the initiatives taken as part of the Autonomic Communications Forum. We remain however convinced that the future of autonomics in the networking sphere will focus and be successful only if applied on well identified and limited control loops like it was done here for the renumbering function. A further step is to assess the autonomic infrastructure against malicious behavior.

\section{ACKNOWLEDGEMENT}

The work reported in this paper was partially supported by the Cisco CARD collaborative initiatives program. The latest developments of the NDPMon toolkit were partially supported by the FP6 IST-EMANICS Network of Excellence.

We would also like also to thank Fred Baker and Tony Hain for their valuable feedback.

\section{REFERENCES}

[1] S. Deering and R. Hinden, "Internet Protocol, Version 6 (IPv6) Specification," RFC 2460 (Draft Standard), Dec. 1998.

[2] T. Narten, E. Nordmark, and W. Simpson, "Neighbor Discovery for IP Version 6 (IPv6)," RFC 2461 (Draft Standard), Dec.1998

[3] S. Thomson and T. Narten, "IPv6 Stateless Address Autoconfiguration," RFC 2462 (Draft Standard), Dec. 1998.

[4] F. Baker, E. Lear, and R. Droms. Procedures for Renumbering an IPv6 Network without a Flag Day RFC 4192 (Informational), Sept. 2005

[5] P. Nikander, J. Kempf, and E. Nordmark, "IPv6 Neighbor Discovery (ND) Trust Models and Threats," RFC 3756 (Informational), May 2004.

[6] R. Enns, Ed., "NETCONF Configuration Protocol”, RFC 4741 (Standards Tracks), Dec. 2006.

[7] S. Deering, "ICMP Router Discovery Messages" RFC 1256, Sep. 1991

[8] D.C. Plummer, "An Ethernet Address Resolution Protocol” RFC 826 (STD37), Nov. 1982.

[9] IBM White Paper. "An Architectural blueprint for autonomic computing”, June 2005.

[10] B. Carpenter, R. Atkinson and H. Flinck, « Renumbering still needs work », draft work in progress, May 2009.

[11] E. Hughes and A. Somayaji « Towards Network Awareness », Proc. LISA'2005

[12] R. Hinden, S. Deering, «IP Version 6 Addressing Architecture » RFC 4291, (Draft Standard), Feb 2006.

Frederic Beck holds a M. Sc. in Networking and Telecommunication degree from Université Louis Pasteur in Strasbourg. After working at Alcatel as an R\&D engineer, he joined INRIA in Nancy as a Research Engineer in Dec. 2004. His research interests are mainly centered in IPv6 (transition, renumbering and formerly multicast and mobility) as well as network supervision and security.

Isabelle Chrisment is a Professor in Computer Science at ESIAL (Ecole Supérieure d'Informatique et Applications de Lorraine), Nancy University (France). She received her $\mathrm{PhD}$ in Computer Science in 1996 from the University of Nice-Sophia Antipolis and her Habilitation Degree in 2005 from Nancy University. Since 2002, she is vice-head of the MADYNES team, LORIA-INRIA Lorraine whose research domain is the management of dynamic aspects provided by networks and services. Her research area is related to network configuration and security.

Dr. Ralph Droms is a Cisco Distinguished Engineer in the Research and Advanced Development group, where he focuses on name and address management, IPv6 and development of protocol standards for Smart Grid. He supports and participates in research collaborations such as protocol modeling, automated methods for IPv6 deployment and renumbering and IPv6 mobile ad hoc networks. He has participated in the IETF for many years, having organized the DHC working group in 1989, which he chaired until he was selected to be an Internet Area Director for the IETF in 2009. Prior to joining Cisco, Dr. Droms was on the faculty at Bucknell and Penn State. He has also been on the research staff at both IBM and Burroughs (Unisys). Dr. Droms is a co-author of "The DHCP Handbook". His PhD is in computer science from Purdue University.

Olivier Festor is a research director at INRIA Nancy-Grand Est where he leads the MADYNES research team. He has a Ph.D. degree (1994) and an Habilitation degree (2001) from Henri-Poincare University, Nancy, France. He spent 3 years at the IBM European Networking Center in Heidelberg, Germany and one year at the EURECOM Institute in Nice, France. His research interests are in the design of algorithms and models for automated security management of large scale networks and services. This includes monitoring, fuzzing and vulnerability assessment. Application domains are IPv6, Voice over IP services and dynamic ad-hoc networks.

He has published more than 70 papers in network and service management and serves in the technical program and organization committees as well as in the editorial boards of several international conferences and journals. He was the TPC Co-chair of the IFIP/IEEE IM'2005 event. Since 2006, he leading the EMANICS European Network of Excellence dedicated to Management Solutions for the Future Internet and was named co-chair of the IFIP TC6 Working Group 6.6 co-chair in 2008. 